\title{
Seroprevalence of rubella in the cord blood of pregnant women and congenital rubella incidence in Nha Trang, Vietnam
}

Masami Miyakawa ${ }^{\mathrm{a}, 1}$, Hiroshi Yoshino ${ }^{\mathrm{b}}$, Lay Myint Yoshida ${ }^{\mathrm{b}}$, Emilia Vynnycky,f, Hideki Motomura ${ }^{\mathrm{a}}$, Le Huu Tho ${ }^{\mathrm{d}}$, Vu Dinh Thiem ${ }^{\mathrm{e}}$, Koya Ariyoshi ${ }^{\mathrm{b}}$, Dang Duc Anh ${ }^{\mathrm{e}}$, and Hiroyuki Moriuchi ${ }^{\mathrm{a}^{*}}$

a Department of Molecular Microbiology and Immunology, Graduate School of Biomedical Sciences, Nagasaki University, 1-7-1 Sakamoto, Nagasaki 852-8501, Japan.

${ }^{b}$ Department of Clinical Medicine, Institute of Tropical Medicine, Nagasaki University, Sakamoto, Nagasaki, Japan

${ }^{c}$ Modelling \& Economics Unit, Public Health England, Colindale Ave, London, Greater London, UK

${ }^{d}$ Khanh Hoa Provincial Public Health Service, Nha Trang, Vietnam

${ }^{e}$ National Institute of Hygiene and Epidemiology, Yecxanh, Hai Ba Trung, Ha Noi, Vietnam

${ }^{f}$ Department of Epidemiology and Population Health, London School of Hygiene \& Tropical Medicine, Keppel Street, London, England

$<$ Footnotes $>$

*Corresponding author. Tel: +81 95819 7297; fax: +81 958197301.

E-mail addresses: masami5008@gmail.com (M. Miyakawa), hiromori@nagasaki-u.ac.jp (H. Moriuchi). 
${ }^{1}$ Present address: Bureau of International Medical Cooperation Japan, National Center for Global Health and Medicine, 1-21-1, Toyama Shinjuku-ku, Tokyo, Japan. Tel.: +81 332027181. 


\begin{abstract}
To investigate susceptibility to and factors associated with rubella infection among pregnant mothers and to estimate the burden of congenital rubella infection (CRI) in Vietnam where rubella-containing vaccine (RCV) is not included in the routine immunization programme, we conducted a prospective cohort study in Nha Trang, Vietnam between 2009 and 2010. Rubella-specific immunoglobulin-M and immunoglobulin-G were investigated in cord blood samples by enzyme immunoassay. Corresponding clinical-epidemiological data were analyzed and the national congenital rubella syndrome (CRS) incidence was estimated using modeling. We enrolled 1988 pairs of mothers aged 17-45 years and their newborn babies. No mothers had received RCV. Multivariate analysis revealed that mothers aged 17-24 (aOR 2.5, 95\% CI: 1.7-3.8) or 25-34 (1.4, 1.0-2.1) years were more likely to be susceptible than those aged 35-45 years. Overall 28.9\% (574/1988, 95\% CI: 26.9-30.9\%) of mothers were seronegative. The CRI rate was 151 (95\% CI: 0-322) per 100,000 live births. Modeling estimated that 3788 babies (95\% CI: 3283-4143) were born with CRS annually in Vietnam with an overall CRS incidence of 234 (95\% CI: 207-262) cases per 100,000 live births. A substantial proportion of women of childbearing age (WCBA) are at risk of rubella infection during pregnancy and this can result in a high frequency of miscarriage or burden of CRS across Vietnam. Prompt introduction of RCV into national immunization programme with catch-up vaccination to children and WCBA will reduce CRI in Vietnam.
\end{abstract}

Keywords: incidence; seroprevalence; women of childbearing age; congenital rubella syndrome; cord blood; IgG; IgM; modeling 


\section{Introduction}

Rubella is a mild self-limiting infection associated with fever and rash and mainly occurring in children. However, if infected in their first 8-10 weeks of gestation, up to $90 \%$ of pregnant women may face miscarriage, stillbirth or a child born with congenital rubella syndrome (CRS). This is associated with significant disability, including sensorineural deafness, cataract, and cardiac defects [1-3]. Therefore the primary goal in controlling rubella is to prevent miscarriage and CRS by an adequate rubella immunization programme [2].

The epidemiology and burden of CRS in Vietnam is poorly understood. The number of WHO member countries which had introduced rubella-containing vaccine (RCV) into their routine immunization programme increased from 83 (43\%) in 1996 to 131 (68\%) in 2010, resulting in an 82\% decline in the number of reported rubella cases by 2009 [4,5]. However, out of 36 countries in the Western Pacific Region, Vietnam remains one of 6 countries which have not used RCV as of 2009, and has been experiencing periodic rubella outbreaks and, potentially, a large burden of CRS. Adolescents and adults comprised most of the reported cases of rubella in outbreaks, and, notably, $60 \%$ of female cases were WCBA in 2009 [6,7].

Due to under-reporting of rubella cases and difficulties with diagnosing CRS, estimates of the incidence of rubella and burden of CRS obtained from surveillance data are often unreliable. However, adequate population-based serological data collected through a prospective cohort study may provide insight into these statistics. Such data have not been available in Vietnam before 2012.

In this paper, we describe a prospective cohort study of rubella serological survey in Nha Trang, Central Vietnam which aimed to 1) investigate susceptibility to and factors associated with rubella infection among pregnant mothers, 2) investigate the rate and outcomes of congenital rubella infection (CRI), and 3) estimate the burden of CRS in Vietnam. 


\section{Materials and Methods}

\subsection{Study Design, Period and Location}

A prospective cohort study was conducted from May 2009 through May 2010 in Nha Trang. This study site has a population of approximately 390,000 and is located in Khanh Hoa Province, Central Vietnam. Khanh Hoa General Hospital (KHGH) is a provincial hospital with 900 beds which covers the whole Khanh Hoa Province, and provides comprehensive medical services, with approximately 6000 babies delivered annually. The study was conducted in 16 out of 24 communes in Nha Trang because detailed population and demographic data were available for these communes from our census surveys in 2006 and 2010. Since Nha Trang is a tourist city, communes which have big blocks of government administrative buildings, hotels and restaurant areas were not included in the survey. RCV has not been introduced into the national immunization programme at the time of the study. A negligible proportion of the population is believed to get access to RCV at the private sector/clinics in Nha Trang, although private sector vaccination services have been expanding in Hanoi and Ho Chi Minh City [7].

\subsection{Subjects and Recruitment Process}

The samples were collected as part of a birth cohort study. Mothers aged $\geq 17$ years residing in 16 target communes in Nha Trang who delivered a single child at KHGH without severe complications on weekdays during this study period were recruited, together with their newborn babies. The study recruited pregnant women with no serious complications on admission during the study period because it is unethical or difficult to ask a pregnant mother who is in a serious condition on admission for informed consent. We did not recruit women who give birth to two or more babies in the study because the CRI rate of the babies may differ and it will be difficult to analyze the risk factors. We did not collect samples during weekends due to a shortage of staff, but recorded all the deliveries, including those on weekends, to calculate the CRI incidence. Written informed consent was obtained from participants, and clinicalepidemiological information was collected through interview using a structured questionnaire before the 
delivery and from medical charts or maternal health cards. The whole process was done by two trained research nurses and supervised by a research clinician.

\subsection{Sample Collection and Testing}

Cord blood samples were collected at the time of delivery. The blood was centrifuged for 5 minutes at $1500 \mathrm{rpm}$, and separated plasma was kept in $-80^{\circ} \mathrm{C}$ freezers. The plasma samples were transported to Nagasaki, Japan [8], and tested for rubella-specific Immunoglobulin-M (IgM) and Immunoglobulin-G (IgG) by enzyme immunoassay (EIA) (Mini VIDAS). The cut-off values of the assay were 1.2 and 4.0, respectively, in accordance with the company recommendation.

\subsection{Characteristic Categorization Definition}

Maternal educational level was categorized into three groups, based on the educational system in Vietnam, namely none-primary, lower-upper secondary and higher education, which were defined as 0-5, 6-12, and $\geq 13$ years of schooling, respectively [9]. Paternal occupations were classified into four groups: 1 , unemployed (including students); 2, unskilled workers/farmers/soldiers; 3, officers/skilled workers; and 4, professionals/owners of enterprise. Residential areas where the mothers lived were defined as urban and rural according to Vietnam government administrative categorization. The number of mothers who visited antenatal care $(\mathrm{ANC}) \geq 4$ times was calculated, following WHO recommendations [10]. Body mass index (BMI) was defined as the weight in kilograms divided by the square of the height in meters $\left(\mathrm{kg} / \mathrm{m}^{2}\right)$ and underweight was classified as BMI $<18.5$ by WHO criteria [11]. Anemia was defined for pregnant women according to WHO criteria as a haemoglobin of $<11.0 \mathrm{~g} / \mathrm{dl}$ [12]. Births were considered preterm if they occurred after less than 37 gestational weeks (GWs), and low birth weight (LBW) if the birth weight (BW) was less than $2500 \mathrm{~g}$, following the guidelines in the International Classification of Diseases-10: version 2010 [13]. A diagram of BW percentiles by GWs was created based on data collected from subjects, using previous methods [14,15], with a mean BW at 40 weeks of $3379.6 \mathrm{~g}$ and a standard deviation of $11.4 \%$ of the mean BW. Babies who were "light-for-dates" (defined as those with BW below 
the $10^{\text {th }}$ percentile [13]) were identified from this figure (Appendix 1). CRI was defined using the WHO case definition [15] as infection with no clinical signs of CRS but positive for rubella-specific IgM.

\subsection{Data Management, Statistical Analysis}

All the collected information was managed confidentially throughout the process. The data were double-entered and cleaned, and statistical analysis was conducted with IBM SPSS Statistics 18. Odds ratio (OR) or adjusted OR (aOR) with its 95\% confidence interval (95\% CI) and p-values for factors associated with seronegativity were calculated by either chi-square or logistic regression for univariate analysis and by logistic regression for multivariate analyses. A p-value of less than 0.05 was considered statistically significant. The overall proportion of individuals who were seronegative based on rubellaspecific IgG and the proportion who were rubella-specific IgM-positive were calculated together with their 95\% CI. The age distribution of the enrolled mothers and the age-specific proportion that were seronegative were plotted. Infants with CRI were invited for medical follow-up including physical, neurodevelopment and auditory examinations at 10 months and 24 months of age. Auditory function tests were conducted with distortion product otoacoustic emission for the former session and automated auditory brainstem response for the latter (echo-screen II). Furthermore, cardiovascular examination was conducted with echocardiogram (Viamo SSA-640A) for the latter session. Japanese pediatricians performed the above examinations in cooperation with Vietnamese staff.

\subsection{Estimating the CRS Incidence using Modeling}

Following previous methods [16], an age-structured catalytic model was fitted to the observed agestratified serological data using maximum likelihood to estimate the force of infection for rubella (defined as the rate at which susceptible are infected per unit time). The force of infection was assumed to differ between the age groups $<13$ years and $\geq 13$ years. The best-fitting value for the force of infection was then used to estimate the CRS incidence per 100,000 live births among women in 5 year age groups, aged between 15 and 44 years using the equation $0.65 \times s(A)(1-\exp (-16 \lambda \mathrm{o} / 52)) \times 100,000$, where $s(A)$ is the 
proportion of women in age group $A$ that are susceptible and $\lambda_{o}$ is the force of infection among those aged over 13 years. The CRS incidence per 100,000 live births among mothers aged 15-44 was calculated as the average of the CRS incidence per 100,000 live births in each five-year maternal age group, weighted by the number of live births in the corresponding maternal age group in 2009 .

As in previous analyses [16], the risk that a child was born with CRS was assumed to be $65 \%$ if the mother was infected during the first 16 weeks of pregnancy and zero thereafter. The CRS incidence per live birth among women in each age group was then multiplied by estimates of the number of live births (including those occurring at week-ends) occurring among women in the corresponding age group in 2009, in these areas to obtain the number of CRS cases born in the five-year age group. The number of live births was calculated by multiplying the age-specific fertility rates [17] by the age-specific number of females in each age group in 2009, as estimated in the national census conducted in the year 2010 [18].

Confidence intervals on the force of infection and CRS incidence were obtained by profile likelihood.

\subsection{Ethical Consideration}

This study was approved by the Ethical Committees of Nagasaki University (Japan) and National Institute of Hygiene and Epidemiology (Vietnam).

\section{Results}

\section{Recruitment Profile}

During the study period, 3173 deliveries by mothers living in 16 target communes in Nha Trang were reported in total. Of them, 2787 (87.8\%) occurred at KHGH and 1988 (62.7\%) mothers who fitted the recruitment criteria were enrolled for this study.

Characteristics of the enrolled participants 
The mean age of the enrolled mothers was 27.9 years, ranging from 17 to 45 years with the ages following normal distribution (Figure 1). Over $85 \%$ of mothers had received lower secondary or higher education, and nearly all fathers had some occupation. Seventy percent of mothers were urban residents. About 50\% of mothers were primiparous, and nearly $85 \%$ had visited antenatal clinics at least 4 times for this pregnancy. Over a quarter of mothers had a low BMI and around a quarter were anemic. As many as $41.8 \%$ of all births were delivered by caesarean section regardless of the underlying reason. Mean GWs at deliveries were 39.1 weeks with $3.4 \%$ being preterm. The male-to-female ratio was 51.6 : 48.4. Their mean BW was 3262.7 g with 2.3\% LBW and 10.9\% "light-for-date” (Table 1). None of the mothers had been vaccinated with RCV.

\section{Factors Associated with Seronegativity}

Overall, 28.9\% (574/1988 [95\% CI: 26.9-30.9\%]) of mothers were seronegative with an increased proportion (44.3\% [95\% CI: 34.3-54.4\%]) of mothers aged 17-19 years being seronegative (Table 2). Young age, primipara, increased number of ANC visits, and preterm delivery were significantly associated with maternal seronegativity in univariate analyses, while in multivariate analyses the only significant factors were young age (aOR2.5 [95\% CI: 1.7-3.8] and 1.4 [95\% CI: 1.0-2.1] for 17-24 and 2534 year olds, respectively, compared to those aged 35-45 years), and increased number of ANC visits (aOR1.7 [95\% CI: 1.2-2.3] for those with $\geq 4$ ANC visits, compared with those with $<4$ visits) (Table 1)

\section{Rubella-specific IgM-positive participants}

Three infants were positive for rubella-specific IgM among 1988 study subjects; therefore, the CRI rate in this study was 151 (95\% CI: 0-322) per 100,000 live births. Their mothers lived in different residential areas, and no rubella epidemic had been reported in those areas during the study period. None of them had apparent abnormalities in physical examinations at birth. Two of them were evaluated at 10 months and 24 months of age and showed no abnormalities in physical examinations, neurodevelopmental tests, echocardiography, and auditory function tests. The parent of the remaining child reported in a telephone 
interview that he had been well without any apparent delay in psychomotor or language development, although we could not examine him because his family had moved outside the study area.

\section{Model-based estimates of the CRS Incidence}

The best-fitting value for the force of infection was about 5.3\%/year (95\% CI: 4.7-5.8\%) for children aged $<13$ years and about 3.9\%/year (95\% CI: 3.5-4.4\%/year) for those aged $\geq 13$ years (log likelihood

deviance: 28, 21df) (Figure 2). The estimated CRS incidence was about 234 per 100,000 live births among 15-44 year olds, and decreased with increasing age from 348 per 100,000 births among women aged 15-19 years to 130 per 100,000 live births among those aged 40-44 years (Table 2).

After combining these figures with data on the number of live births, these estimates suggest that 3788 babies (95\% CI: 3283-4143) were born with CRS in Vietnam in 2009, with 24 (95\% CI: 21-26), 36 (95\% CI: 31-39) and 49 (95\% CI: 42-53) born in the 16 study communes, Nha Trang City and Khanh Hoa Province, respectively.

\section{Discussion}

This study is the first population-based large-scale seroprevalence study among pregnant women in Vietnam elucidating the risk of rubella infection for WCBA in this country. Overall, a very high proportion (28.9\%) of WCBA were found to be susceptible, suggesting that the incidence of CRS per 100,000 live births was also very high (234 per 100,000 live births) with over 3000 cases of CRS born each year.

The only previous data relating to rubella seroprevalence in Vietnam considered pregnant Vietnamese emigrants in Taiwan and Australia [19, 20]. Our finding that 28.9\% of pregnant women were susceptible to rubella is consistent with that from these reports, in which $24-36 \%$ of pregnant women were susceptible 
[19, 21]. In another study investigating 45 developing countries before RCV had been used, the susceptibility in WCBA was generally $15-20 \%$ but exceeded 25\% in 12 countries [22]. ${ }^{5,5}$

Multivariate analysis revealed that young maternal age was associated with seronegativity. After multivariate analysis including all other possible confounding factors in the model aside from age, aOR for age still remained over 1.0, which indicates that it can be an independent factor for increased risk of rubella infection.

Our multivariate analysis supported no association of parity or maternal educational status with rubella susceptibility, which is consistent with findings elsewhere [23-26]. Apparent association on univariate analysis was probably due to increased maternal age as a confounding factor, although there might be a potential risk of transmission from elder siblings to mothers.

Association of residential area, e.g. rural or urban, with seropositivity has also varied between studies. For example, some studies conducted before rubella vaccination was introduced found lower levels of seropositivity in rural than in urban areas [27], one recent study found the converse [25], while no association was identified elsewhere $[23,26]$ as seen from this study. Rubella-specific IgM tests identified three infants with CRI, which implies a CRI rate of 151 (95\% CI: 0-322) per 100,000 live births. This low incidence rate is consistent with the fact that no epidemic had been reported during the study period. None of these babies had apparent abnormalities, confirming that there are more individuals with CRI than with clinically apparent CRS.

We estimated that on average 3788 (95\% CI: 3283-4143) infants with CRS would be born annually in Vietnam with 234 (95\% CI: 207-262) cases per 100,000 live births. This estimate is not significantly different from that in a study at the National Obstetric Hospital, Hanoi, where the incidence of CRS was 210 per 100,000 live births [28]. Differences between the model predictions and the reported number of CRS cases may be attributed to several factors, including under-reporting, resulting from problems with 
diagnosing CRS in infants, and the fact that the estimate does not account for abortions which may have occurred once pregnant mothers became infected.

This study has several limitations. First of all, this study only considered seroprevalence in Nha Trang, located in central Vietnam. This area is perhaps more rural than other parts of Vietnam and the findings may not be easily generalizable to other areas. However, since our study covered the majority of deliveries in this area, our results are probably generalizable to similar regions in Vietnam. Second, we could have introduced selection bias by restricting recruitment to women who lived in 16 out of 24 communes in Nha Trang and delivered on weekdays. But excluding the samples on weekends should not have any significant effect on the CRS incidence calculated using by modeling, since the calculations used the age-specific proportion of women who were seronegative and the total number of deliveries, including those occurring on weekends. Thirdly, the present study considered the seroprevalence in women aged 17-45 years but had no data from younger ages. Seroprevalence data from all age groups should provide improved insight into the epidemiology of rubella and extent of ongoing transmission in Vietnam. Finally, following WHO recommendations, we used EIA for rubella-specific IgM when diagnosing CRS cases [8], although this has a low positive predictive value in low transmission settings and lower sensitivity, compared to the virus genome detection by reverse transcription polymerase chain reaction (RT-PCR). However, due to logistical and technical constraints, RT-PCR was not used.

The high proportion (28.9\%) of WCBA that were found to be susceptible and the high burden of CRS predicted by modeling suggest that there is a need to introduce immunization with RCV into Vietnam.

Since females aged 17-24 years are at increased risk of giving birth to infants with CRS, adolescent girls should be targeted for RCV immunization to prevent CRS, as described previously [29, 30]. To do so, as many opportunities as possible would need to be exploited, including supplementary immunization activities carried out for measles, visits for children’s immunization, and postpartum check-up, as suggested previously $[6,7,31]$. School-based programs for girls, similar to those conducted elsewhere in 
the past [29] may be feasible in Vietnam, where $80 \%$ of girls attend secondary school [32]. Furthermore, since the current national programme to give 3 doses of tetanus toxoid targets to women aged 15-35 years in high-risk areas, extension of this programme to other regions will provide further opportunities for RCV immunization [7].

In addition, coverage of measles vaccination is quite high in Vietnam, providing favorable conditions for adding RCV in the Expanded Program of Immunization (EPI) for children. Two doses of measles vaccine for children at age 9 and 18 months can be replaced by Measles-Rubella or Measles-MumpsRubella vaccine in the revised EPI, the cost-effectiveness of which has been estimated previously [6]. However, as found elsewhere and suggested by modeling [2, 29, 34, 35], introducing RCV into childhood EPI at coverage levels which are too low can lead to adverse increases in the proportion of women that are susceptible to infection and the incidence of CRS. Therefore, a catch-up campaign targeting all children aged 13 years or younger and vaccinating WCBA would need to be introduced RCV is introduced into the routine schedule.

Finally, establishment of CRS surveillance is recommended, so that the actual burden of CRS can be directly measured and to help assess the effectiveness of the revised preventive interventions [2, 31].

In conclusion, a substantial proportion of WCBA are at risk of rubella infection during pregnancy, potentially resulting in a large burden of miscarriage or CRS in Vietnam. Prompt introduction of RCV to WCBA is strongly recommended for averting such adverse outcomes. In order to eliminate rubella, this would need to be followed by a catch-up campaign among children and incorporation of RCV into EPI.

\section{Funding}


This work was supported by Japan Initiative for Global Research Network on Infectious Diseases (JGRID) and Japan Society for the Promotion of Science (Grants-in-Aid for Scientific Research [grant number 21406028]).

\section{Acknowledgments}

This was a collaborated research among Nagasaki University (Japan), NIHE (Vietnam), and Khanh Hoa Provincial Public Health Service (Vietnam). We would like to express our gratitude to all participants and those who cooperated for this study in both Vietnam and Japan.

Conflict of interest: None declared. 


\section{References}

[1] Wilbert HM. Chapter 244 Rubella. Nelson Textbook of Pediatrics. 18th ed. Philadelphia: Saunders; 2007.

[2] WHO. Rubella vaccines: WHO position paper. Geneva: World Health Organization; Wkly Epidemiol Rec 2011;86:301-16. Available from: http://www.who.int/entity/wer/2011/wer8629.pdf [accessed 20 June 2012].

[3] Miller E, Cradock-Watson JE, Pollock TM. Consequences of confirmed maternal rubella at successive stages of pregnancy Lancet 1982;2:781-4.

[4] CDC. Progress toward control of rubella and prevention of congenital rubella syndrome --worldwide, 2009. Centers for Disease Control and Prevention (CDC); Morb Mortal Wkly Rep (MMWR) 2010; 59:1307-10. Available from: http://www.cdc.gov/mmwr/pdf/wk/mm5940.pdf [accessed 20 June 2012].

[5] Countries Using Rubella Vaccine in National Immunization Schedule, 2010. WHO/IVB database, Data as of Sep 2011. World Health Organization 2011. Available from: http://www.who.int/immunization_monitoring/diseases/Rubella_map_schedule.jpg [accessed 20 June 2012].

[6] WHO. Report: Eighteenth Meeting of the Technical Advisory Group on Immunization and Vaccine Preventable Diseases in the Western Pacific Region2009, Manila, Philippines. Regional Office for the Western Pacific: World Health Organization 2009.

[7] Review of Expanded Program of Immunization Vietnam 2009, National EPI Review Report. Viet Nam: United Nations Children's Fund 2009. Available from: http://www.unicef.org/vietnam/EPI_NATIONAL_Review_Report_Vietnam_2009_Final.pdf [accessed 20 June 2012]. 
[8] WHO. Manual for the laboratory diagnosis of measles and rubella virus infection, Second edition, Geneva: World Health Organization2007. Available from: http://www.who.int/immunization_monitoring/LabManualFinal.pdf [accessed 20 June 2012].

[9] Education Landscape. Ministry of Education and Training, Vietnam 2006. Available from: http://en.moet.gov.vn/?page=6.7\&view=3401 [accessed 20 June 2012].

[10] WHO. Pregnancy, childbirth, postpartum and newborn care: a guide for essential practice. Geneva: World Health Organization2006. Available from: http://whqlibdoc.who.int/publications/2006/924159084X_eng.pdf [accessed 20 June 2012].

[11] BMI classification, Global Database on Body Mass Index. World Health Organization 2012. Available from: http://apps.who.int/bmi/index.jsp?introPage=intro_3.html [accessed 20 June 2012].

[12] UNICEF/UNU/WHO.Iron deficiency anaemia: assessment, prevention, and control. A guide for programme managers. UNICEF/UNU/WHO 2001. Available from: http://whqlibdoc.who.int/hq/2001/WHO_NHD_01.3.pdf [accessed 20 June 2012].

[13] ICD-10 Version: 2010. World Health Organization 2010. Available from: http://apps.who.int/classifications/icd10/browse/2010/en [accessed 20 June 2012].

[14] Mikolajczyk RT, Zhang J, Betran AP, et al. A global reference for fetal-weight and birthweight percentiles. Lancet 2011; 377: 1855-61.

[15] WHO. WHO-recommended standards for surveillance of selected vaccine-preventable diseases. World Health Organization 2003. Available from: http://www.who.int/vaccinesdocuments/DocsPDF06/843.pdf [accessed 20 June 2012].

[16] Cutts FT, Vynnycky E. Modelling the incidence of congenital rubella syndrome in developing countries. Int J Epidemiol 1999; 28: 1176-84.

[17] World Fertility Data 2008.United Nations Population Division/DESA Fertility and Family Planning Section. Available from: http://www.un.org/esa/population/publications/WFD\%202008/WP_WFD_2008/Data.html 
[18] The 2009 Vietnam Population and Housing Census. Department for Population and Labor Statistics, Hanoi 2010

[19] Tseng HF, Chang CK, Tan HF, et al. Seroepidemiology study of rubella antibodies among pregnant women from seven Asian countries: evaluation of the rubella vaccination program in Taiwan. Vaccine 2006; 24: 5772-7.

[20] Francis BH, Thomas AK, McCarty CA. The impact of rubella immunization on the serological status of women of childbearing age: a retrospective longitudinal study in Melbourne, Australia. Am J Public Health 2003; 93: 1274-6.

[21] Lin CC, Yang CY, Shih CT, et al. Rubella seroepidemiology and catch-up immunization among pregnant women in Taiwan: comparison between women born in Taiwan and immigrants from six countries in Asia. Am J Trop Med Hyg 2010; 82: 40-4.

[22] Cutts FT, Robertson SE, Diaz-Ortega JL, et al. Control of rubella and congenital rubella syndrome (CRS) in developing countries, Part 1: Burden of disease from CRS. Bull World Health Organ 1997; 75: 55-68.

[23] Palihawadana P, Wickremasinghe AR, Perera J. Seroprevalence of rubella antibodies among pregnant females in Sri Lanka. Southeast Asian J Trop Med Public Health 2003; 34: 398-404.

[24] Dayan GH, Panero MS, Urquiza A, et al. Rubella and measles seroprevalence among women of childbearing age, Argentina, 2002 Epidemiol Infect 2005; 133: 861-9.

[25] Sasmaz T, Kurt AO, Ozturk C, et al. Rubella seroprevalence in women in the reproductive period, Mersin, Turkey. Vaccine 2007; 25: 912-7.

[26] Pehlivan E, Karaoglu L, Ozen M, et al. Rubella seroprevalence in an unvaccinated pregnant population in Malatya, Turkey. Public Health 2007; 121: 462-8.

[27] Dowdle WR, Ferrera W, De Salles Gomes LF et al. WHO collaborative study on the seroepidemiology of rubella in Caribbean and Middle and South American populations in 1968. Bull World Health Organ 1970; 42: 419-422. 
[28] Medical Doctor Thesis, Hanoi Medical School, 2012: Several factors affect the CRS at the National OB Hospital in Hanoi.

[29] Robertson SE, Cutts FT, Samuel R, et al. Control of rubella and congenital rubella syndrome (CRS) in developing countries, Part 2: Vaccination against rubella. Bull World Health Organ 1997; 75: 6980.

[30] WHO. Report of a meeting on preventing congenital rubella syndrome: immunization strategies, surveillance needs. Geneva: World Health Organization 2000. Available from: http://www.who.int/vaccines-documents/DocsPDF00/www508.pdf [accessed 20 June 2012].

[31] WHO. Western Pacific Regional Guidelines: Introducing Rubella Vaccine, draft of 25 Mar 2004. Regional Office for the Western Pacific: World Health Organization 2004.

[32] At a glance: Vietnam, Statistics. United Nations Children's Fund. Available from: http://www.unicef.org/infobycountry/vietnam_statistics.html [accessed 20 June 2012].

[33] Hinman AR, Irons B, Lewis M, et al. Economic analyses of rubella and rubella vaccines: a global review. Bull World Health Organ 2002; 80: 264-70.

[34] Panagiotopoulos T, Antoniadou I, Valassi-Adam E. Increase in congenital rubella occurrence after immunisation in Greece: retrospective survey and systematic review. BMJ 1999; 319: 1462-7.

[35] Vynnycky E, Gay NJ, Cutts FT. The predicted impact of private sector MMR vaccination on the burden of Congenital Rubella Syndrome. Vaccine 2003; 21: 2708-19. 
Table 1. Demographic characteristics and factors associated with seronegativity for rubella-specific IgG, Nha Trang, Vietnam, 2009-2010

\begin{tabular}{|c|c|c|c|c|}
\hline \multirow[t]{2}{*}{ Factors } & All & IgG(-) & $\begin{array}{c}\text { Univariate } \\
\text { Analysis }\end{array}$ & $\begin{array}{c}\text { Multivariate } \\
\text { Analysis }\end{array}$ \\
\hline & $\mathbf{N}(\%)$ & N (\%) & OR (95\% CI) & aOR (95\% CI) \\
\hline \multicolumn{5}{|c|}{ 【Mothers】 } \\
\hline \multicolumn{5}{|l|}{ Maternal Age (in years) ${ }^{b}$} \\
\hline $17-24$ & $572(28.8)$ & $213(37.2)$ & $2.5^{\mathrm{a}}(1.8,3.7)$ & $2.5(1.7,3.8)$ \\
\hline $25-34$ & $1166(58.7)$ & $313(26.8)$ & $1.6(1.1,2.2)$ & $1.4(1.0,2.1)$ \\
\hline $35-44$ & 249 (12.5) & 47 (18.9) & Ref & Ref \\
\hline 45 & $1(0.05)$ & $1(100)$ & - & - \\
\hline \multicolumn{5}{|l|}{ Maternal Education ${ }^{\mathrm{b}}$} \\
\hline None-Primary & 249 (12.5) & $58(23.3)$ & $0.7(0.5,1.0)$ & $0.9(0.6,1.3)$ \\
\hline Lower-Upper Secondary & $1240(62.4)$ & 367 (29.6) & $1.0(0.8,1.2)$ & $1.0(0.8,1.3)$ \\
\hline Higher & $499(25.1)$ & 149 (29.9) & Ref & Ref \\
\hline \multicolumn{5}{|l|}{ Paternal Occupation } \\
\hline Unemployed & $6(0.3)$ & $3(50.0)$ & $2.1(0.4,10.5)$ & $1.6(0.3,8.3)$ \\
\hline Unskilled Workers/Farmers/ Soldiers & $820(41.6)$ & $232(28.3)$ & $0.8(0.6,1.1)$ & $0.9(0.6,1.2)$ \\
\hline Officers/Skilled Workers & $887(45.0)$ & $252(28.4)$ & $0.8(0.6,1.1)$ & $0.8(0.6,1.1)$ \\
\hline Professionals/Owners of Enterprise & $259(13.1)$ & $84(32.4)$ & Ref & Ref \\
\hline \multicolumn{5}{|l|}{ Residential Area } \\
\hline Suburban & $590(29.7)$ & $182(30.9)$ & $1.1(0.9,1.4)$ & $1.2(0.9,1.4)$ \\
\hline Urban & $1398(70.3)$ & $392(28.0)$ & Ref & Ref \\
\hline \multicolumn{5}{|l|}{ Para } \\
\hline Primipara & $998(50.2)$ & 318 (31.9) & $1.3(1.1,1.6)$ & $1.0(0.8,1.3)$ \\
\hline Multipara & $990(49.8)$ & 256 (25.9) & Ref & Ref \\
\hline \multicolumn{5}{|l|}{$\mathrm{N}$ of ANC Visit } \\
\hline$\geq 4$ times & $1684(84.7)$ & $511(30.3)$ & $1.7(1.2,2.2)$ & $1.7(1.2,2.3)$ \\
\hline$<4$ times & 304 (15.3) & $63(20.7)$ & Ref & Ref \\
\hline \multicolumn{5}{|l|}{ Maternal BMI } \\
\hline Low & 514 (25.9) & $162(31.5)$ & $1.2(1.0,1.5)$ & $1.1(0.8,1.3)$ \\
\hline Normal-High & $1474(74.1)$ & $412(28.0)$ & Ref & Ref \\
\hline \multicolumn{5}{|l|}{ Anemia } \\
\hline Yes & $508(25.6)$ & 135 (26.6) & $0.9(0.7,1.1)$ & $0.9(0.7,1.1)$ \\
\hline
\end{tabular}


No

Mode of Delivery

Cesarean Section

Vaginal Delivery

Preterm Delivery

Yes

No

\begin{tabular}{ccccc} 
No & $1921(96.6)$ & $563(29.3)$ & Ref & Ref \\
LBW & \multicolumn{2}{c}{ 【Neonates】 } \\
Yes & $45(2.3)$ & $9(20)$ & $0.6(0.3,1.3)$ & $0.8(0.3,1.8)$ \\
No & $1943(97.7)$ & $565(29.1)$ & Ref & Ref \\
Light-for-Date & & & \\
Yes & $217(10.9)$ & $61(28.1)$ & $1.0(0.7,1.3)$ & $1.0(0.7,1.4)$ \\
No & $1771(89.1)$ & $513(29.0)$ & Ref & Ref \\
Sex & & & & \\
Boys & $1026(51.6)$ & $312(30.4)$ & $1.2(1.0,1.4)$ & $1.2(1.0,1.4)$ \\
Girls & $962(48.4)$ & $262(27.2)$ & Ref & Ref \\
\hline
\end{tabular}

atems with $p$-value $<0.05$ with statistical significance were underlined.

${ }^{b}$ Logistic regression was used for univariate analysis for the indicated factors and for multivariate analysis, while chi's square analysis was utilized for univariate analysis for the remaining factors.

N, number; OR, odds ratio; CI, confidence interval; aOR, adjusted OR; ANC, antenatal care; BMI, body mass index; and Ref, reference 
Table 2. Age-specific percentage of seronegative individuals, estimates of the CRS incidence per 100,000 live births, and the number of CRS cases born in the year 2009 in Vietnam

\begin{tabular}{|c|c|c|c|c|}
\hline \multirow[b]{2}{*}{$\begin{array}{l}\text { Age group } \\
\text { (in years) }\end{array}$} & \multicolumn{2}{|c|}{ Results of this study } & \multicolumn{2}{|c|}{ Estimates by modeling } \\
\hline & $\begin{array}{c}\text { All } \\
\text { N (\%) }\end{array}$ & $\begin{array}{c}\text { IgG (-) } \\
\text { N (\%) (95\%CI) }\end{array}$ & $\begin{array}{c}\text { CRS incidence per } \\
100,000 \text { live births } \\
(95 \% \mathrm{CI})\end{array}$ & $\begin{array}{c}\text { CRS cases in } 2009 \\
\text { in Vietnam } \\
\mathrm{N}(95 \% \mathrm{CI})\end{array}$ \\
\hline $17-19$ & $97(4.9)$ & $43(44.3)(34.3,54.4)$ & $348(308,389)^{\mathrm{a}}$ & $254(225,284)^{\mathrm{a}}$ \\
\hline $20-24$ & 475 (23.9) & $170(35.8)(31.5,40.1)$ & $285(253,319)$ & $1091(967,1221)$ \\
\hline $25-29$ & $678(34.1)$ & $181(26.7)(23.4,30.0)$ & $234(208,262)$ & $1514(1343,1694)$ \\
\hline $30-34$ & $488(24.5)$ & $132(27.0)(23.1,31.0)$ & $192(170,215)$ & $599(531,670)$ \\
\hline $35-39$ & 207 (10.4) & $44(21.3)(15.6,26.9)$ & $158(140,177)$ & $259(188,238)$ \\
\hline $40-44$ & $42(2.1)$ & $3(7.1)(0.0,15.3)$ & $130(115,145)$ & $71(29,36)$ \\
\hline 45 & $1(0.1)$ & $1(100)(-)$ & - & - \\
\hline Total & $1988(100)$ & $574(28.9)(26.9,30.9)$ & $234(207,262)^{b}$ & $3788(3283,4143)$ \\
\hline
\end{tabular}

${ }^{a}$ The numbers in parentheses reflect 95\% CI, based on profile likelihood.

${ }^{\mathrm{b}}$ Calculated as the average of the CRS incidence in five-year maternal age groups for 15-44 year olds, weighted by the number of live births in each age group.

$\mathrm{N}$, number; $\mathrm{CI}$, confidence interval 


\section{Figure legends}

Figure 1. Frequency distribution of the ages of enrolled participants, Nha Trang, Vietnam, 2009-2010.

The solid line shows the curve for the normal distribution.

Figure 2. Comparison between predictions of the age-specific percentage individuals who were seronegative (solid line), obtained using the best-fitting catalytic model, and the observed data, Nha Trang, Vietnam, 2009-2010 (crosses, with 95\% CI).

Appendix 1. Birth weight percentiles of study participants by gestational week, Nha Trang, Vietnam, 2009-2010.

A diagram of birth weight percentiles by gestational week was created based on the data of subjects with a calculator proposed previously, assuming a mean birth weight at 40 weeks of $3379.6 \mathrm{~g}$ of and a standard deviation of $11.4 \%$ of the mean birth weight. Light-for-dates, defined as those below the $10^{\text {th }}$ percentile of birth weight, were identified from this figure. 
Fig.1

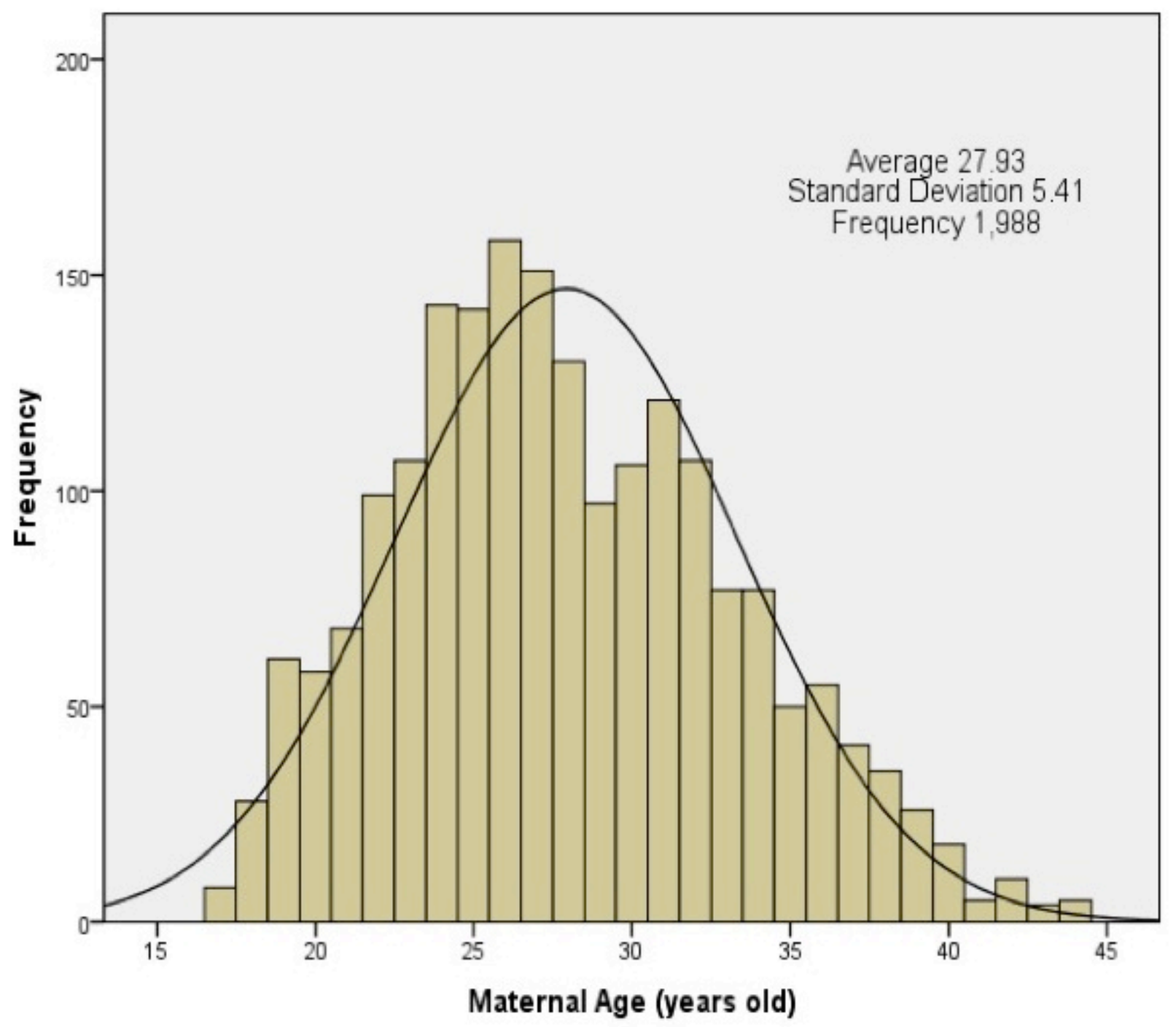




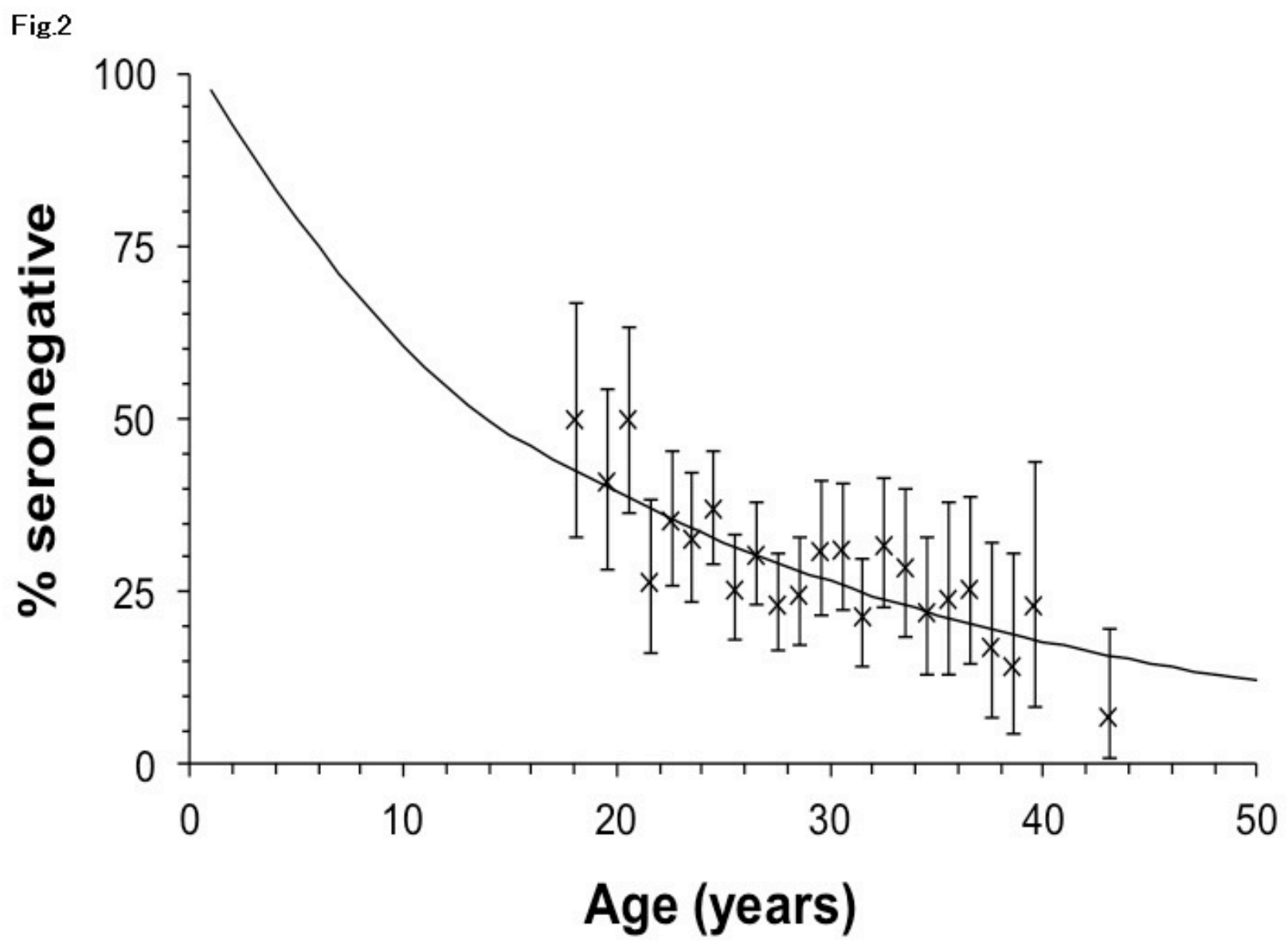




\section{Appendix 1.}

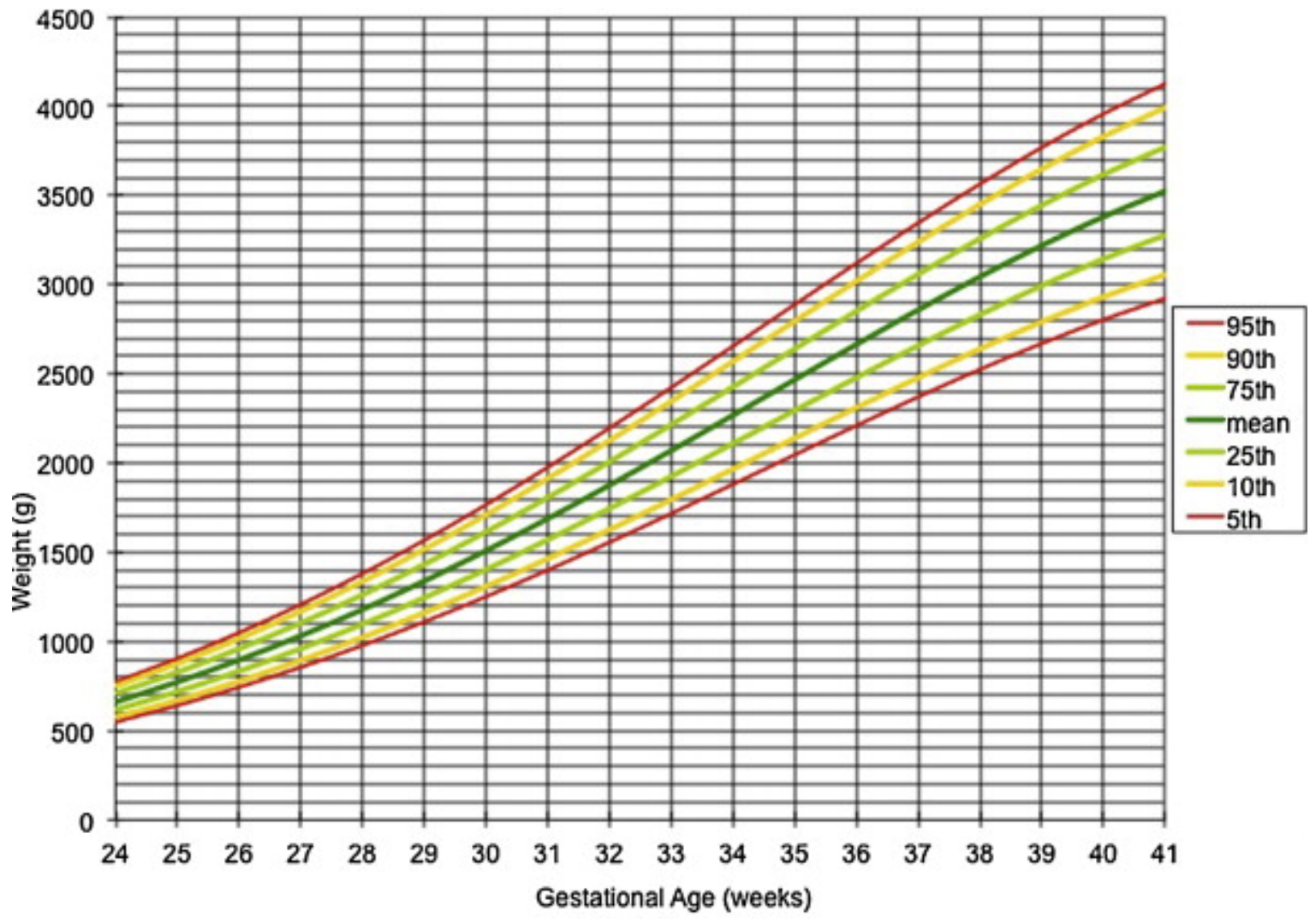

